\title{
Atuação do enfermeiro brasileiro no ambiente escolar: Revisão narrativa
}

\author{
Performance of brazilian nurses in the school environment: Narrative review \\ Desempeño de las enfermeras brasileñas en el entorno escolar: Revisión narrativa
}

\author{
Patrícia de Oliveira Bastos \\ ORCID: https://orcid.org/0000-0002-7052-8017 \\ Universidade Estadual do Ceará, Brasil \\ E-mail: paty.bastos@aluno.uece.br \\ José Jader Moreira Junior \\ ORCID: https://orcid.org/0000-0002-8770-8954 \\ Centro Universitário Ateneu, Brasil \\ E-mail: jjadermj@ahoo.com.br \\ Maria Emanuela Silveira Norjosa \\ ORCID: https://orcid.org/0000-0001-9056-2127 \\ Centro Universitário Ateneu, Brasil \\ E-mail: mariamanusilveira80@gmail.com \\ Maria Joelma Carneiro Vasconcelos \\ ORCID: https://orcid.org/0000-0003-4625-2521 \\ Centro Universitário Ateneu, Brasil \\ E-mail: joelmavasc16@gmail.com \\ Maisa Leitão de Queiroz \\ ORCID: https://orcid.org/0000-0002-9465-3402 \\ Conselho Nacional de Pesquisa, Brasil \\ E-mail: q.1.maisa@gmail.com
}

\begin{abstract}
Resumo
Introdução: O contexto escolar representa um espaço estratégico para aquisição de conhecimentos, habilidades, mudanças de comportamento e estilos de vidas, por ser um local em que o educando desenvolve vínculos e permanece, geralmente, por um tempo prolongado. Objetivo: Descrever as evidências de estudos sobre a atuação do enfermeiro brasileiro no ambiente escolar. Método: Trata-se de uma revisão narrativa da literatura. As buscas foram desenvolvidas entre os meses de abril e maio de 2020, nas bases de dados: Literatura Latino-Americana e do Caribe em Ciências da Saúde (Lilacs), Scientific Electronic Library Online (Scielo), Banco de Dados em Enfermagem Bibliografia Brasileira (BDEnf) via Biblioteca Virtual em Saúde (BVS) e na literatura cinzenta. Utilizou-se os Descritores de Ciências da Saúde (DeCS) e as palavras-chave relacionadas à temática: Enfermagem escolar, Saúde escolar, Promoção da saúde, School nursing, School health e Health promotion. A análise crítica dos estudos e a síntese dos dados bibliográficos foram realizadas de forma descritiva, agrupados por temas pertinentes ao assunto estudado. Resultados: Os resultados encontrados permitiram apresentar os comentários em três grandes categorias temáticas, sendo essas: A escola como ambiente de intervenção do enfermeiro para formação integral e saudável; A significativa contribuição do enfermeiro no ambiente escolar brasileiro e a Influência da educação e promoção da saúde no ambiente escolar. Considerações finais: Percebeu-se que a atuação do enfermeiro no ambiente escolar é fundamental para o desenvolvimento e crescimento dos alunos e ainda a promoção da saúde de todos os atores envolvidos no ambiente escolar.
\end{abstract}

Palavras-chave: Enfermagem escolar; Saúde escolar; Promoção da saúde.

\begin{abstract}
Introduction: The school context represents a strategic space for the acquisition of knowledge, skills, changes in behavior and lifestyles, as it is a place where the student develops bonds and usually stays for a long time. Objective: To describe the evidence from studies on the role of Brazilian nurses in the school environment. Method: This is a narrative review of the literature. The searches were carried out between April and May 2020, in the following databases: Latin American and Caribbean Literature on Health Sciences (Lilacs), Scientific Electronic Library Online (Scielo), Database on Nursing Brazilian Bibliography (BDEnf) via the Virtual Health Library (VHL) and in gray literature. The Health Sciences Descriptors (DeCS) and the keywords related to the theme were used: School nursing, School health, Health promotion, School nursing, School health and Health promotion. The critical analysis of the studies and the synthesis of bibliographic data were carried out in a descriptive way, grouped by themes relevant to the studied subject. Results: The results found allowed the comments to be presented in three major thematic categories, namely: The school as an environment for nurses' intervention for comprehensive and healthy education; The significant contribution of nurses in the Brazilian school environment and the Influence of education and health promotion in the school environment. Final considerations: It was noticed that the role of nurses in the school
\end{abstract}


environment is essential for the development and growth of students and also the health promotion of all actors involved in the school environment.

Keywords: School nursing; School health; Health promotion.

\begin{abstract}
Resumen
Introducción: El contexto escolar representa un espacio estratégico para la adquisición de conocimientos, habilidades, cambios de comportamiento y estilos de vida, ya que es un lugar donde el alumno desarrolla vínculos y habitualmente permanece por mucho tiempo. Objetivo: Describir la evidencia de estudios sobre el papel de las enfermeras brasileñas en el ámbito escolar. Método: Se trata de una revisión narrativa de la literatura. Las búsquedas se realizaron entre abril y mayo de 2020, en las siguientes bases de datos: Literatura Latinoamericana y del Caribe en Ciencias de la Salud (Lilas), Biblioteca Electrónica Científica en Línea (Scielo), Base de Datos de Bibliografía Brasileña de Enfermería (BDEnf) vía Biblioteca Virtual en Salud (BVS) y en la literatura gris. Se utilizaron los Descriptores de Ciencias de la Salud (DeCS) y las palabras clave relacionadas con el tema: Enfermería escolar, Salud escolar, Promoción de la salud, Enfermería escolar, Salud escolar y Promoción de la salud. El análisis crítico de los estudios y la síntesis de datos bibliográficos se realizaron de forma descriptiva, agrupados por temas relevantes para el tema estudiado. Resultados: Los resultados encontrados permitieron presentar los comentarios en tres grandes categorías temáticas, a saber: La escuela como entorno de intervención del enfermero para la educación integral y saludable; La contribución significativa de las enfermeras en el entorno escolar brasileño y la Influencia de la educación y la promoción de la salud en el entorno escolar. Consideraciones finales: Se advirtió que el rol de las enfermeras en el ambiente escolar es fundamental para el desarrollo y crecimiento de los estudiantes y también para la promoción de la salud de todos los actores involucrados en el ambiente escolar.
\end{abstract}

Palabras-clave: Enfermería escolar; Salud escolar; Promoción de la salud.

\title{
1. Introdução
}

O contexto escolar representa um espaço estratégico para aquisição de conhecimentos, habilidades, mudanças de comportamento e estilos de vidas, por ser um local em que o educando desenvolve vínculos e permanece, geralmente, por um tempo prolongado. Este elo entre saúde e educação se relaciona a uma população mais saudável e empoderada das possibilidades do seu cuidado em saúde (Casemiro; Fonseca \& Secco, 2014; Figueiredo; Machado \& Abreu, 2010; Zuge et al., 2020).

No Brasil, os primeiros registros das atribuições do trabalho da enfermeira escolar e da especialização nesta área foram encontrados em 1930. Neste mesmo período, também há o registro da criação do primeiro curso de Higiene Escolar, na Faculdade de Medicina e Cirurgia de São Paulo. Estes marcos sinalizaram as contrapartidas do Brasil para que a educação em saúde começasse a ser reconhecida, valorizada e considerada como aliada à preservação da saúde da população (Pires et al., 2012).

No ano de 2007, pelo decreto presidencial N ${ }^{\circ}$ 6286, foi instituído o Programa Saúde na Escola (PSE), com orientações para promover saúde e educação integral para crianças e jovens. Este vem contribuir para o fortalecimento de ações na perspectiva do desenvolvimento integral e proporcionar à comunidade escolar a participação em programas e projetos que articulem saúde e educação, para o enfrentamento das vulnerabilidades que comprometem o pleno desenvolvimento de crianças, adolescentes e jovens brasileiros. Possui ainda a característica de reconhecer e acolher as ações de integração entre saúde e educação já existentes e que têm impactado positivamente na qualidade de vida dos educandos (Brasil, 2012).

A escola saudável é aquela que possui um ambiente solidário e propício ao aprendizado, por isso ela deve estar engajada no desenvolvimento de políticas públicas saudáveis e na estimulação da criação de entornos favorecedores à saúde, na aprendizagem de comportamentos que permitam a proteção do meio ambiente, na conservação de recursos naturais e na implicação cada vez maior da população em projetos de promoção da saúde (Aerts et al., 2004; Vieira et al., 2017).

Nesse contexto, o profissional de enfermagem é de suma importância na educação escolar, por se utilizar de técnicas lúdicas e saber abordar determinadas temáticas que envolvam tanto as crianças como os adolescentes, propagando assim, a promoção em saúde; deste modo, permitindo-lhes confrontar-se positivamente consigo mesmas, construir um projeto de vida e ser capazes de fazer escolhas individuais, conscientes e responsáveis. Nesse sentido, na fase escolar é comum à pessoa obter 
diversas dúvidas em relação a certos aspectos concernentes a saúde, por isso, o enfermeiro é o profissional capacitado para preencher esses espaços e integrar de modo amplo a estes aspectos (Silva; Reis \& Greinert, 2016; Viner et al., 2020).

Considerando os expostos, observamos que o profissional de enfermagem relacionado a promoção em saúde no âmbito escolar é essencial para a formação crítico, reflexiva do cidadão e o cuidado de si. Assim, o enfermeiro escolar é colocado como desencadeador das ações em saúde, proporcionando a criação de espaços de educação em saúde na escola ressaltando os princípios norteadores da promoção e seus valores éticos como: a vida, a solidariedade, a equidade e a cidadania e uma série de estratégias que visam concretizar a cooperação e as parcerias (Rasche \& Santos, 2013; Viner et al., 2020).

As primeiras enfermeiras da escola praticavam o máximo possível de seus conhecimentos e habilidades, assim como as enfermeiras de prática avançada são encarregadas de fazer atualmente (Cowell, 2012). Hoje, os enfermeiros escolares enfrentam questões de financiamento, advocacia, confusão pública do papel e a necessidade de pesquisas baseadas em evidências para apoiar sua posição em ambientes escolares (Wolfe, 2013; Alencar, 2020).

Dessa forma, foi possível identificar a presença de lacunas no que se diz respeito a atuação do enfermeiro na saúde escolar, sendo escassas as ações para promover uma melhor qualidade de vida e até mesmo reduzir a vulnerabilidade e riscos à saúde, considerando a fragilidade e vulnerabilidade das crianças e adolescentes, que estão expostas a qualquer tipo de problemas do cotidiano, do mais simples até os mais complexos relacionados aos modos de viver, habitação, ambiente, educação, lazer, cultura, acesso a bens e serviços essenciais. Desse modo, faz-se necessário o uso de meios para investigar e propagar o conhecimento acerca da temática. Portanto, o presente estudo apresenta o objetivo de descrever as evidências de estudos sobre a atuação do enfermeiro brasileiro no ambiente escolar, colocado esse como desencadeador de ações em saúde, proporcionando assim, a criação de espaços de educação em saúde na escola.

\section{Metodologia}

\section{Tipo de Estudo}

Trata-se de uma revisão narrativa da literatura. De acordo com Rother (2007), são estudos que do ponto de vista teórico ou conceitual realizam uma revisão ampla a fim de descrever e discutir o desenvolvimento ou o "estado da arte". Assim, esta pode contribuir para o debate de determinadas temáticas, levantamento de questões e colaborar para a aquisição e atualização de conhecimentos em um curto espaço de tempo.

\section{Local e Período do Estudo}

As buscas foram desenvolvidas livremente entre os meses de abril e maio de 2020, nas seguintes bases de dados: Literatura Latino-Americana e do Caribe em Ciências da Saúde (Lilacs), Scientific Electronic Library Online (Scielo) e Banco de Dados em Enfermagem Bibliografia Brasileira (BDEnf) via Biblioteca Virtual em Saúde (BVS). Realizou-se ainda, busca na literatura cinzenta, assim como consultas ao Google Escolar, bases de teses e dissertações da Capes e busca manual realizada de forma detalhada nas referências dos artigos selecionados a fim de amplificar a pesquisa.

\section{Seleção dos Estudos}

Para a busca dos artigos utilizou-se termos escolhidos a partir dos Descritores de Ciências da Saúde (DeCS) e as palavras-chave da literatura científica relacionada à temática: Enfermagem escolar, Saúde escolar, Promoção da saúde, School nursing, School health e Health promotion. Incluíram-se as pesquisas independentemente do tipo de estudo, do idioma, ano de publicação e as que contemplassem o trabalho dos enfermeiros na área da saúde do escolar no Brasil. As publicações que abordavam o trabalho de outras profissões no âmbito escolar e aquelas que não responderam o objetivo proposto foram excluídas da revisão. A partir dos critérios de elegibilidade adotados, levantaram-se 21 (vinte e um) artigos. 


\section{Processo de Extração e Análise dos dados}

A análise crítica dos estudos selecionados e a síntese dos dados bibliográficos foram realizadas de forma descritiva, agrupados por temas pertinentes ao assunto estudado para facilitar a compreensão da evolução do conhecimento na área de saúde do escolar, permitindo observar, descrever e classificar os dados, com a intenção de colaborar com o processo de busca da temática principal deste trabalho.

\section{Custos e Financiamento}

Os custos desembolsados ao estudo foram para fins de material de papelaria e internet necessário para seu o desenvolvimento. $\mathrm{O}$ trabalho possuiu financiamento próprio das pesquisadoras.

\section{Resultados e Discussão}

Os resultados encontrados permitiram apresentar os comentários em três grandes categorias temáticas que atenderam as inquietações que guiaram essa pesquisa, sendo essas: A escola como ambiente de intervenção do enfermeiro para formação integral e saudável; A significativa contribuição do enfermeiro no ambiente escolar brasileiro e a Influência da educação e promoção da saúde no ambiente escolar.

\section{A escola como ambiente de intervenção do enfermeiro para formação integral e saudável}

Os artigos selecionados apontaram que a escola deve ser entendida como um espaço de relações, privilegiado para o desenvolvimento crítico e político, que contribui na construção de valores pessoais, crenças, conceitos e maneira de conhecer o mundo e interfere diretamente na produção social e na saúde (Tinoco; Reis \& Freitas, 2014; Rasche \& Santos, 2013; Gubert et al., 2009; Zuge et al., 2020). No entanto, foi possível observar nos artigos estudados a existência de lacunas referentes à propagação da educação em saúde na escola, deixando fragmentada essa parte de promoção da saúde, focando na maioria dos casos na parte assistencial (Pereira et al., 2013; Alencar, 2020).

Em sua abordagem de pesquisa sobre o Bullying, Coelho e Mendes (2011) constaram que é possível que esse fenômeno seja atenuado pelo profissional de enfermagem, onde a intervenção do enfermeiro, integrado numa equipe multidisciplinar, assume particular importância. Salienta-se que relação equânime estabelecida com a criança/família/comunidade, permite ao enfermeiro que ele se torne um elemento chave na deteç̧ão precoce de situações que possam afetar negativamente a saúde da criança e a sua qualidade de vida, tal como acontece quando esse se encontra envolvido em situações de violência escolar.

Neste ambiente, compreende-se que o enfermeiro é essencial por acrescentar o componente 'saúde' a equipe profissional da escola. Assim, contribui ativamente para a formação crítico, reflexiva do cidadão e para autonomia do cuidado de si, impactando no desenvolvimento humano de forma individual e coletiva (Sousa; Erdmann \& Mochel, 2010; Alencar, 2020).

A partir das análises dos estudos selecionados, observou-se a ênfase dada aos cinco componentes principais do Programa Saúde na Escola (PSE), sendo esses: avaliação clínica e psicossocial, ações de promoção de saúde e prevenção de doenças e agravos, educação permanente e capacitação de profissionais da educação e saúde e de jovens para o PSE, monitoramento e avaliação da saúde dos estudantes e monitoramento e avaliação do PSE (Brasil, 2012).

Por meio dos componentes do PSE o enfermeiro tem a possibilidade de exercer todas as ações descritas em parceria com uma equipe multidisciplinar, devendo esse participar do planejamento e execução de atividades, avaliação nutricional dos escolares, também da coleta de dados antropométricos, como peso, altura, cálculo e análise de IMC (Brasil, 2012).

Em seu estudo Lopes e colaboradores (2007) enfatizam que a falta de um profissional de saúde permanentemente no 
ambiente escolar pode ser vista como um problema, já que a inserção do mesmo neste ambiente reforça a realização de práticas de promoção à saúde e prevenção de doenças possibilitando o cuidado de crianças e jovens em consonância com a união de saberes entre a saúde e educação.

Tal afirmação evidencia que a presença do profissional de enfermagem no ambiente escolar é um fator determinante para que se consiga promover a saúde dos escolares por meio de discussões interativas e técnicas, com os profissionais da educação (Sousa; Espiridião \& Medina, 2017; Viner et al., 2020). E assim, fortalecer as atividades de promoção da saúde e métodos preventivos acerca de agravos em saúde na escola, detectando problemas e possíveis soluções mais rapidamente (Rasche \& Santos, 2013).

\section{A significativa contribuição do enfermeiro no ambiente escolar brasileiro}

Segundo o dispositivo que dispõe sobre o exercício profissional, a Lei n ${ }^{\circ} 7.498$, de 25 de junho de 1986, o artigo 11, inciso II, alínea j, determina que é função privativa do enfermeiro a educação que vise à melhoria na condição de saúde da população (Cofen, 1986). A educação em saúde precisa ser disseminada, incentivando constantemente a adoção de comportamentos seguros e saudáveis.

Quanto da abordagem e conduta do enfermeiro escolar, este é tido como o principal articulador e pilar para a implementação de ações de saúde, como promoção de palestras, rodas de conversas, atividades lúdicas, que envolvam cunho preventivo (Valério, 2015; Viner et al., 2020; Khattab et al., 2020). Ainda de acordo com os mesmos autores, o enfermeiro deve estar principalmente presente nas ações de: profilaxia; controle de doenças; acompanhamento de medidas antropométricas dos alunos; controle de condições sanitárias e aulas de higiene. Souza et al. (2017) expõem a importância da utilização de jogos para interagir com os alunos e mediar o ensino sobre a saúde.

Nos artigos selecionados percebeu-se que o enfermeiro exerce um papel relevante diante da população escolar, pois a prática de atividades e programas voltados para a educação em saúde, adequado as características de saúde do sujeito, família e localidade sistematiza e otimiza as formas como esse profissional pode atuar visando a promoção de saúde (Rasche \& Santos, 2013).

Segundo Gubert et al. (2009), Coelho e Mendes (2011) o trabalho do enfermeiro na escola não deve focar e ser realizado através de ações pontuais, esse deve buscar as verdadeiras necessidades e aspirações dos educandos, favorecendo a criação do vínculo entre enfermeiro e escolares, o que proporciona um atendimento mais qualificado e a quebra barreiras existentes nesse processo.

Assim, a presença do enfermeiro na escola se torna necessária e determinante para que a atenção seja voltada para os processos de promoção da saúde do escolar através de discussões com toda a comunidade, atividades de educação em saúde, estimulação de debates técnicos e da apresentação de sua perspectiva em relação aos processos de saúde e doença nos escolares. Este torna-se responsável pelo cuidado e observação da rotina e do ambiente escolar, atentando para os problemas encontrados e suas possíveis soluções (Rasche \& Santos, 2013).

Em sua pesquisa, Figueiredo et al (2016) relatam que nenhuma das escolas participantes de seu estudo possuíam ou tinham outro profissional da saúde atuante. Seu resultado aponta a importância do enfermeiro em unidades escolares devido ter demonstrado que $45(96 \%)$ dos educadores participantes do seu estudo terem justificado e relatado a presença desse profissional na escola como colaborador nas ações voltadas à saúde, desde a resolução de problemas assistenciais às ações de educação, promoção e prevenção. Os autores afirmaram ainda, que o enfermeiro é um profissional capacitado para auxiliar e informar todos aqueles que compõem o ambiente escolar.

Viu-se ainda, que o alcance de resultados satisfatórios no ambiente brasileiro escolar se relaciona com a articulação da escola com as esquipes de saúde, esta deve ser pautada nos interesses dos usuários e ser capaz de satisfazer suas necessidades 
de saúde. Por meio da participação em um meio escolar articulado o profissional de enfermagem podem realizar açães voltadas para a atenção à saúde, por meio das quais se torna possível a formação de cidadãos sensibilizados acerca de hábitos de vida saudáveis (Coelho \& Mendes, 2011; Tinoco; Reis \& Freitas, 2014)

Machado et al., (2011) numa intervenção voltada para escola, descreveu que a equipe de enfermagem se mostrou como agente de promoção do desenvolvimento e crescimento infantil, numa perspectiva de qualidade de vida, auxiliou na construção de hábitos saudáveis e de uma cultura de paz, além de promover habilidades para resolução de problemas pessoais e na comunidade.

Assim vemos que a atuação do enfermeiro como educador é extremamente fundamental, pois vai além das práticas educativas e assistenciais. Corroborando com essa ideia Rasche e Santos (2013) e o PSE descrevem que esse profissional deve: promover, estimular, integrar e desenvolver atividades que de forma direta ou indireta se relacionem com a saúde dos escolares (Viner et al., 2020).

\section{A influência da educação e promoção da saúde no ambiente escolar}

O Ministério da Saúde (MS) compreende que a escola como é um dos pontos chaves para se trabalhar a saúde na perspectiva da promoção da saúde (Pereira et al., 2013). Dado que segundo o MS a promoção da saúde é uma das estratégias de produção de saúde, ou seja, como um modo de pensar e de operar articulado às demais políticas e tecnologias desenvolvidas pelo sistema de saúde brasileiro, contribuem para a elaboração de ações que respondam às necessidades sociais em saúde (Brasil, 2014). Sendo essa então, compreendida como uma estratégia de articulação transversal, integrada e intersetorial (Costa et al., 2013).

Os artigos mostram que por meio da educação em saúde as pessoas são capazes de desenvolver autonomia e responsabilidade perante sua própria saúde, sendo essa uma prática social crítica e transformadora utilizada na prevenção de agravos (Gubert et al., 2009; Zuge et al., 2020). Nesse contexto, os profissionais de enfermagem se destacam por ter a ação educativa como um de seus principais eixos norteadores para o cuidado. Portanto, a possibilidade de praticar o cuidado de enfermagem no ambiente escolar possibilita a formação de atitudes e valores, pelos escolares e todos aqueles que compõem a escola, em conformidade com comportamentos saudáveis e que resultem em benefícios individuais e coletivos (Tinoco; Reis; Freitas, 2014; Viner et al., 2020).

Machado et al., (2011) salienta que a educação em saúde tem sido conceituada como uma proposta de aprendizagem que tem como finalidade principal facilitar o desenvolvimento de ações que conduzam a uma melhoria do estado de saúde. Para tanto, ela pretende desenvolver no indivíduo e/ou grupo capacidades e habilidade para analisar criticamente a sua realidade, contribuindo para a autoformação do indivíduo e para tomada de decisões. Bem como a pesquisas de Lima et al., (2012) que apontaram que a promoção da saúde no espaço escolar é relevante devido promover a propagação efetiva de informações em saúde na rede de ensino.

Dessa forma, as ações de promoção da saúde podem promover um olhar ao indivíduo e ao seu contexto social, possibilitando o acesso às informações sobre os malefícios do uso do álcool e drogas, incentivo a um aporte nutricional adequado, oferta de métodos contraceptivos, orientações sobre a sexualidade, a prática de atividades físicas e lazer para um bom desenvolvimento físico, emocional, intelectual e social. Essas ações englobam uma escuta diferenciada e focada nos problemas deste grupo, direcionando, assim, uma assistência embasada na promoção e prevenção de agravos (Gubert et al., 2009; Figueiredo et al., 2016; Viner et al., 2020).

\section{Considerações Finais}

Verificou-se que ainda são escassas as produções científicas sobre a atuação do enfermeiro no ambiente escolar, devido se perceber a necessidade do preenchimento de lacunas de conhecimentos acerca da temática e notou-se que poucas 
pesquisas foram desenvolvidas na área da enfermagem com o intuito de testar intervenções.

Percebeu-se que a atuação do enfermeiro no ambiente escolar é fundamental para o desenvolvimento e crescimento dos alunos e ainda a promoção da saúde de todos os atores envolvidos no ambiente escolar. O perfil de cuidador e educador do enfermeiro faz com que ele se sobressaia nos espaços pedagógicos, por facilitar a disseminação de informações verídicas sobre saúde e bem-estar.

Salienta-se ainda, que os escolares seguem nessa fase de descobrimento de si, sendo indispensável nesse contexto a atuação do enfermeiro como mediador entre o conhecimento sobre saúde e o aluno como detentor de questionamentos a serem solucionados, contribuindo assim, para o desenvolvimento hábitos saudáveis e para o cuidado de si.

Dessa forma, torna-se importante que o profissional de enfermagem tenha a sensibilidade de reconhecer a escola como um espaço de grande importância para a promoção da saúde, devido esse possuir um papel primordial na formação de um cidadão crítico capaz de adotar atitudes que promovam sua própria qualidade de vida, de todos da escola, da sociedade e de sua família.

Assim, destaca-se a necessidade da realização de novos estudos com ênfase no processo da atuação do enfermeiro no ambiente escolar, com o objetivo de fortalecer e aumentar os campos de atuação dos serviços de enfermagem, contribuir significativamente para a promoção da saúde do escolar e gerar informações e conhecimento para sociedade sobre a importância e necessidade do profissional de enfermagem no âmbito escolar.

\section{Referências}

Aerts, D., Alves, G. G., La Salvia, M. W., \& Abegg, C. (2004). Promoção de saúde: a convergência entre as propostas da vigilância da saúde e da escola cidadã. Cadernos de Saúde Pública, 20, 1020-1028.

Alencar, N. E. S. (2020). A práxis da enfermagem escolar no contexto da pandemia pelo novo coronavírus. Revista Interdisciplinar, 13.

BRASIL. Ministério da Saúde. Política Nacional de Promoção da Saúde - PNaPS: revisão da Portaria MS/GM nº 687, de 30 de março de 2006. Brasília - DF; 2014.

BRASIL. Ministério da Saúde. Secretaria de Atenção à Saúde. Programa Saúde na Escola.

Casemiro, J. P., Fonseca, A. B. C. D., \& Secco, F. V. M. (2014). Promover saúde na escola: reflexões a partir de uma revisão sobre saúde escolar na América Latina. Ciência \& saúde coletiva, 19, 829-840.

Coelho, S. M. S., \& Mendes, I. M. D. M. (2011). Da pesquisa à prática de enfermagem aplicando o modelo de adaptação de Roy. Escola Anna Nery, 15, 845850 .

COFEN. Conselho Federal de Enfermagem. Lei 7.498/86, de 25 de junho de 1986. Dispõe sobre a regulamentação do exercício da enfermagem e dá outras providencias [Internet]. Brasília, DF: Cofen; 1986.

Costa, G. M. C., Cavalcanti, V. M., Barbosa, M. L., Celino, S. D. D. M., França, I. S. X. D., \& Sousa, F. S. D. (2013). Promoção de saúde nas escolas na perspectiva de professores do ensino fundamental.

de Lima, K. Y. N., de Oliveira Moreira, P. N., Tourinho, F. S. V., \& Santos, V. E. P. (2014). Assistência de enfermagem ao adolescente no âmbito escolar: uma pesquisa documental. Revista Enfermagem UERJ, 22(2), 226-232.

do Amaral Tinoco, V., Reis, M. M. T., \& Freitas, L. N. (2014). O enfermeiro promovendo saúde como educador escolar: atuando em primeiros socorros. Revista Transformar, 1(6), 104-113.

Figueiredo, T. A. M. D., Machado, V. L. T., \& Abreu, M. M. S. D. (2010). A saúde na escola: um breve resgate histórico. Ciência \& Saúde Coletiva, 15, 397402 .

Gubert, F. D. A., Santos, A. C. L. D., Aragão, K. A., Pereira, D. C. R., Vieira, N. F. C., \& Pinheiro, P. N. D. C. (2009). Tecnologias educativas no contexto escolar: estratégia de educação em saúde em escola pública de Fortaleza-CE. Rev. eletrônica enferm, 165-172.

Khattab, N., Abbas, A., Abbas, A. R., \& Memon, S. F. (2020). Children returning to schools following COVID-19: A balance of probabilities-Letter to the Editor. International Journal of Surgery (London, England), 79, 202.

Leandro da Silva, A., Carvalho dos Reis, E., \& Milhorine Greinert, B. R. (2016, December). Atuação do enfermeiro na promoção da saúde escolar. In Congresso Brasileiro Interdisciplinar na Promoção da Saúde.

Lee, J. (2020). Efeitos sobre a saúde mental do fechamento de escolas durante o COVID-19. The Lancet Child \& Adolescent Health, 4 (6), 421. 
Research, Society and Development, v. 10, n. 9, e31410918089, 2021

(CC BY 4.0) | ISSN 2525-3409 | DOI: http://dx.doi.org/10.33448/rsd-v10i9.18089

Lopes, G. T., Bernardes, M. M. R., Acauan, L. V., Felipe, I. C. V., Casanova, E. G., \& Lemos, B. K. D. J. (2007). O enfermeiro no ensino fundamental: desafios na prevenção ao consumo de álcool. Escola Anna Nery, 11, 712-716.

Pereira, C. D. F. D., Tourinho, F. S. V., Ribeiro, J. L. D. S., Medeiros, S. B. D., \& Santos, V. E. P. (2013). Padrões funcionais de saúde: diagnósticos de enfermagem em escolares da rede pública. Texto \& Contexto-Enfermagem, 22, 1056-1063.

Pires, L. M., Queirós, P. D. S., Munari, D. B., Melo, C. F. D., \& Souza, M. M. D. (2012). A enfermagem no contexto da saúde do escolar: revisão integrativa da literatura.

Rasche, A. S., \& Santos, M. D. S. S. D. (2013). Enfermagem escolar e sua especialização: uma nova ou antiga atividade. Revista Brasileira de Enfermagem, $66,607-610$.

Rother, E. T. (2007). Revisión sistemática X Revisión narrativa.

Sousa, F. G. M. D., Erdmann, A. L., \& Mochel, E. G. (2010). Modelando a integralidade do cuidado à criança na Atenção Básica de Saúde. Revista Gaúcha de Enfermagem, 31, 701-707.

Sousa, M. C. D., Esperidião, M. A., \& Medina, M. G. (2017). A intersetorialidade no Programa Saúde na Escola: avaliação do processo político-gerencial e das práticas de trabalho. Ciência \& Saúde Coletiva, 22, 1781-1790.

Souza, V. D., Gazzinelli, M. F., Soares, A. N., Fernandes, M. M., Oliveira, R. N. G. D., \& Fonseca, R. M. G. S. D. (2017). O jogo como estratégia para abordagem da sexualidade com adolescentes: reflexões teórico-metodológicas. Revista Brasileira de Enfermagem, 70, 376-383.

Valério, M. (2015). A importância do profissional enfermeiro em uma instituição de educação infantil: um estudo de caso sobre suas atribuições-Escola Municipal de Ensino Fundamental Senador Darcy Ribeiro-Porto Velho/RO.

Vieira, A. G., de Castro Aerts, D. R. G., Câmara, S., Schubert, C., Gedrat, D. C., \& Alves, G. G. (2017). A escola enquanto espaço produtor da saúde de seus alunos. Revista Ibero-Americana de Estudos em Educação, 916-932.

Zuge, B. L., Engers, P. B., Corrêa, S. L. P., Fernandes, T., de Souza, M. B., \& Copetti, J. (2020). Promoção de saúde na educação infantil e anos iniciais: possibilidades e desafios da Base Nacional Comum Curricular. Research, Society and Development, 9(9), e387996634-e387996634. 